\title{
Sistema para classificação de Distúrbios de Qualidade de Energia Elétrica baseado em SVM one class
}

\author{
Bruna de Paula Faria1. Thais Martins Mendes². \\ Danton Diego Ferreira ${ }^{3}$
}

\author{
${ }^{1}$ Departamento de Engenharia - Universidade Federal de Lavras, Lavras - Brasil (UFLA) \\ (Tel: 37 99855-6322; e-mail: bdepaulafaria@hotmail.com) \\ ${ }^{2}$ Departamento de Engenharia Elétrica - Universidade Federal de Juiz de Fora, Juiz de Fora - Brasil (UFJF) \\ (e-mail: martins_tha@outlook.com) \\ ${ }^{3}$ Departamento de Engenharia - Universidade Federal de Lavras, Lavras - Brasil (UFLA), \\ (e-mail:danton@ufla.com.br)
}

\begin{abstract}
Widely, researchers are now turning their attention to Electrical Energy Quality (EEQ), due to unwanted disturbs on electrical signals, caused mostly by the increasing applications of power electronics. This paper introduces a EEQ's classify system. To classify these disturbs was used a One Class Support Vector Machine. Superior Order Statistics (SOS) based parameters were extracted from tension signals to be detected and classified. After that, Fisher discrimination ratio was used to select and pick the fittest parameters. Once they were taken, possibles simplifications are done on algorithm and decent results were achieved.
\end{abstract}

Resumo: Em grande escala a atenção de pesquisadores tem sido direcionada à Qualidade de Energia Elétrica (QEE), devido as indesejadas interferências sofridas pelo sinal elétrico causadas pela ampla e crescente utilização da eletrônica de potência. Este trabalho apresenta um sistema para classificar distúrbios de qualidade de energia elétrica (QEE). Para realização da classificação foi feito uso da chamada Máquina de Vetor de Suporte one class. Parâmetros baseados em Estatística de Ordem Superior (EOS) foram extraídos dos sinais de tensão para serem apresentados ao detector e classificador. A razão de discriminação de Fisher foi aplicada para selecionar os parâmetros mais relevantes. Uma vez obtidos os melhores parâmetros e realizadas as possíveis simplificações no algoritmo de classificação, resultados satisfatórios foram alcançados.

Keywords: Power Quality, Disturbance Detection, Support Vector Machine, Classification of Disturbances.

Palavras-chaves: Qualidade de Energia, Classificação de Distúrbios, Distúrbios Elétricos.

\section{INTRODUÇÃO}

O campo de estudo em qualidade de energia (QEE) compreende diferentes áreas, sejam elas de sistemas de energia elétrica em geral até transmissões de dados em rede. Sua visão acompanha desde geração, transmissão e consumo da mesma, com o objetivo de estudar a qualidade das formas de onda das tensões e correntes elétricas de um sistema. Sua importância gera enorme interesse de pesquisadores, em virtude do crescimento de diferentes aplicações elétricas e as necessidades de atender as novas demandas energéticas da crescente população mundial.

As irregularidades nas formas de onda, são definidas pelo IEC (Commission, 1991) como distúrbios, em destaque os harmônicos, flutuações de tensão, afundamentos, transitórios, entre outros que evidenciam a falta de QEE. Distúrbios são gerados em diferentes eventos nos sistemas de potência, seja em operações normais como chaveamentos de banco de capacitores ou entrada e saída de cargas lineares ou não, enquanto outros eventos não esperados também podem causar influências na QEE, como descargas elétricas atmosféricas e as falhas da própria rede.

Foram realizadas algumas adições às classificações do IEC pela IEEE (IEEE Recommended Practice for Monitoring Electric Power Quality, 2009), com a inclusão de uma nova categoria: distorção de forma de onda da corrente alternada (AC), que abrangia as distorções harmônicas e de componente contínua (DC). Por isso, uma das atribuições da QEE é o monitoramento e análise destes distúrbios ao longo de todo o ciclo do sinal elétrico. Para isso, os sinais de tensão e/ou corrente do sistema monitorado devem ser armazenados para posterior análise ou analisados em tempo real (Ferreira, 2010).

Porém, o armazenamento dessas informações ocuparia grande espaço de disco rígido. Para contornar esse problema são implementados sistemas de detecção que possam armazenar apenas os sinais com distúrbio e, em seguida, fazer a sua análise, classificação e, por fim, determinar a origem das fontes geradoras de distúrbio. Tais sistemas de detecção devem ser de baixa complexidade para que possam processar rapidamente os sinais (Ferreira, 2010). Para isso, são extraídos e analisados determinados parâmetros que passam por um 
algoritmo que identifica e classifica qual tipo de distúrbio está presente nos sinais. Para a extração destes parâmetros, os dados dos sinais são passados para um novo espaço onde as informações necessárias são evidenciadas e comparadas com sinais sem distúrbios para análise das diferenças entre eles.

Dessa forma, o desafio de projetar um detector com baixa complexidade e que apresente o maior desempenho possível, reside na escolha das melhores técnicas para extração dos dados relevantes. Diante deste escopo, esse trabalho propõe um sistema de detecção de distúrbios de QEE que se baseia na utilização de estatísticas de ordem superior (EOS). Esse método foi escolhido em virtude da sua vantagem em apresentar imunidade ruídos gaussianos (Nikias and Petropulu, 1993), e foi, primeiramente, aplicada ao problema de detecção e classificação de distúrbios elétricos em Ribeiro et al. (2007) e Ferreira et al. (2006) que produziram resultados de alto desempenho com reduzida complexidade computacional. A novidade do método proposto está no uso do algoritmo de detecção baseado em máquinas de vetor de suporte (SVM), algoritmo este não supervisionado conhecido como 'SVM one class' (Theodoridis et al., 2010). Seu uso se torna interessante pois ele necessita apenas dos dados de uma das classes de um problema de classificação de duas classes.

\section{PROBLEMA DE DETECÇÃO E CLASSIFICAÇÃO DE DISTÚRBIOS DE QEE}

Para formular o problema em questão, detecção e classificação de distúrbios elétricos, podem ser utilizadas as hipóteses (Ferreira et al., 2014):

$H_{0}: v=f+r$

$H_{1}: v=f_{c}+i+t+h+r$,

Em que $\mathrm{f}=\left[f[n] \ldots f[n-N-1]^{T}, f_{c}=f_{c}[n-N-\right.$ $1]]^{T}, i=[i[n] \ldots i[n-N-1]]^{T}, t=[t[n] \ldots t[n-N-$ $1]]^{T}, h=[h[n] \ldots h[n-N-1]]^{T}, r=[r[n] \ldots r[n-N-$ 1]] ${ }^{T}$ correspondem a $N$ amostras, respectivamente, do sinal fundamental nominal com amplitude e frequência fixas, do sinal fundamental corrompido por alguma variação na frequência ou amplitude, tais como, harmônicos, transitórios e ruídos. A hipótese $H_{0}$ tem relação com a operação nominal do sistema elétrico e a hipótese $H_{1}$ está associada às condições anormais, ou seja, distúrbios no sistema elétrico.

\section{METÓDO PROPOSTO}

O diagrama de blocos da Fig. 1 ilustra a metodologia proposta neste trabalho. Nos itens listados a seguir cada um dos blocos são explicados.

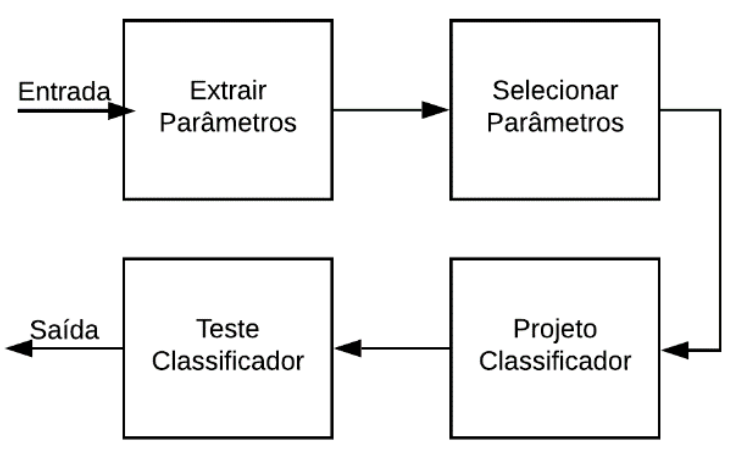

Fig. 1. Método Proposto

\section{DADOS DE ENTRADA}

Foram considerados sinais de tensão sintéticos de seis classes diferentes de distúrbios, sendo elas: afundamentos de tensão, elevação de tensão, harmônicos, transitórios oscilatórios, transitórios impulsivos e notches.

Os sinais foram gerados com uma frequência de amostragem igual a $15.360 \mathrm{~Hz}$ e com um ruído branco Gaussiano aditivo a SNR (Signal-to-noise ratio) de $60 \mathrm{~dB}$. De acordo com (Tomic et al., 2007) o SNR varia entre 50 e $70 \mathrm{~dB}$ no sistema de energia elétrica. A entrada do sistema corresponde a um sinal de tensão com 256 amostras (apenas um ciclo do sistema de $60 \mathrm{~Hz}$ ).

Foram gerados 500 eventos de cada classe de distúrbio abordada e 500 sinais sem distúrbios.

\section{EXTRAÇÃO E SELEÇÃO DE PARÂMETROS}

A etapa de extração de parâmetros baseou-se em EOS, através do cálculo dos cumulantes de segunda, terceira e quarta ordens do sinal monitorado, visando um melhor desempenho na classificação.

O cálculo dos cumulantes de $2^{\mathrm{a}}, 3^{\mathrm{a}}$ e $4^{\mathrm{a}}$ ordens resultaram em uma dimensão total de 640 parâmetros por sinal, sendo equivalente a 128 amostras de segunda ordem, 256 amostras de terceira ordem e 256 amostras de quarta ordem.

As expressões dos cumulantes de segunda, terceira e quarta ordens de sinal aleatório $x[n]$, quando $\mathrm{E}\{x[\mathrm{n}]\}=0$, são, respectivamente (Mendel, 1991):

$$
\begin{aligned}
& C_{2, x}[i]=E\{x[n] x[n+i]\} \\
& C_{3, x}[i]=E\left\{x[n]^{2}[n+i]\right\} \\
& C_{4, x}[i]=E\left\{x[n] x^{3}[n+i]-3 C_{2, x}[i] C_{2, x}[0]\right\}
\end{aligned}
$$

Para um vetor com comprimento finito $(N)$ as aproximações estocásticas oferecem as seguintes (Mendel, 1991):

$$
\begin{aligned}
& \hat{C}_{2, x}[i]=\frac{2}{N} \sum_{n=0}^{\frac{N}{2}-1} x[n] x[n+i] \\
& \hat{C}_{3, x}[i]=\frac{2}{N} \sum_{n=0}^{\frac{N}{2}-1} x[n] x^{2}[n+i] \\
& \hat{C}_{4, x}[i]=\frac{2}{N} \sum_{n=0}^{\frac{N}{2}-1} x[n] x^{3}[n+i]-2 / N^{2} \sum_{n=0}^{N / 2} x[n] x[n+ \\
& i] x^{2}[n]
\end{aligned}
$$


Em que $i=0,1, \ldots,(\mathrm{N} / 2)-1$.

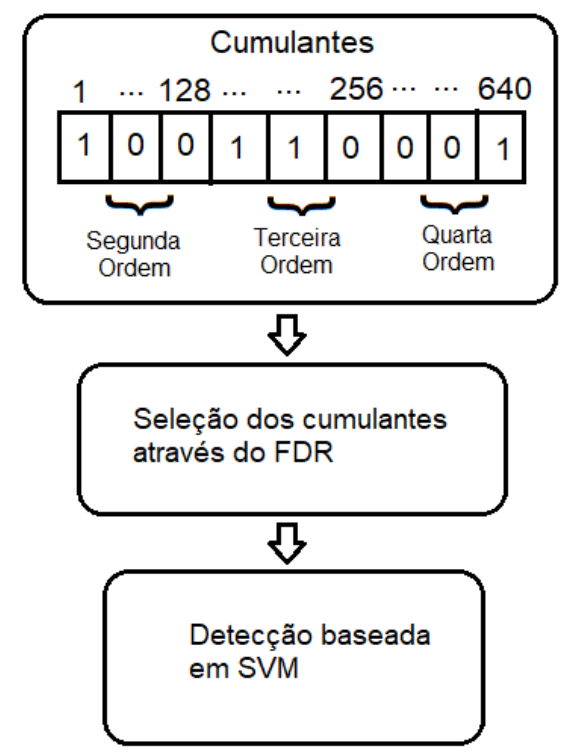

Fig. 2. Seleção dos Cumulantes

Conforme ilustrado na Fig.2, uma vez que os cumulantes tenham sido extraídos, devem-se selecionar aqueles mais representativos de cada classe, ou seja, os que permitem obter uma maior fronteira de separação entre as classes. Assim, decidiu-se usar o FDR (Fisher Discriminat Ratio) pela sua simplicidade e obtenção de resultados satisfatórios [12]. A função custo do critério FDR é:

$$
J_{c}=\left(m_{1}-m_{2}\right)^{T} \odot \frac{1}{\left(D_{1}\right)^{2}+\left(D_{2}\right)^{2}}
$$

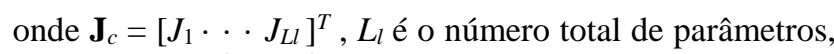
$\mathbf{m}_{1}$ e $\mathbf{m}_{2}$, e $\mathbf{D}_{1}^{2}$ e $\mathbf{D}_{2}^{2}$ são, respectivamente, os vetores de média e variância dos dados da classe 1 e da classe 2. O símbolo $\odot$ refere-se ao produto de Hadamard tal que $r \odot$ $s=\left[\begin{array}{llll}r_{0} s_{0} & \ldots & r_{L r-1} s_{L r-1}\end{array}\right]^{T}$.

Pode-se entender que os i-ésimos elementos do vetor de parâmetros, referentes aos maiores valores de $\mathbf{J}_{c}$, representam os parâmetros que apresentam melhor capacidade de separação entre classes. Esta técnica é bastante útil em processos de classificação mais complexos, uma vez que torna possível selecionar os melhores parâmetros (que mais discriminam as classes envolvidas) e, consequentemente, reduzir o número de parâmetros utilizado na classificação. Esta redução de parâmetros é atraente em sistemas que necessitam de menor complexidade computacional, tais como sistemas que operam em tempo real. Ademais, o uso de um número reduzido de parâmetros pode levar ao projeto de classificadores mais simples e com melhor desempenho, já que os parâmetros responsáveis pela interseção entre classes são, teoricamente, descartados.

\section{ALGORITMO DE DETECÇÃO}

Nesta etapa, aplica-se uma técnica eficiente de detecção de ocorrências, utilizando Máquina de Vetor de Suporte, por meio do processamento dos parâmetros extraídos anteriormente.

Utilizando um conjunto de pontos que possuam duas classes diferentes, o procedimento do SVM trata-se de estabelecer um hiperplano que distribui a maioria dos pontos de mesma classe dispondo-os lado a lado, ao mesmo tempo em que maximiza a distância $(m)$ entre duas classes do presente hiperplano. O hiperplano de separação ótima é aquele em que há a menor distância entre os pontos de uma mesma classe e o hiperplano. Este é traçado pelo SVM ao meio da margem de separação $(m)$. Utilizando o subconjunto de pontos, que demarcam os planos de separação que formam a margem $(m)$, para formar os vetores de suporte (Theodoridis, 2010). É importante ressaltar que, para este trabalho, foi utilizado o kernel RBF (Radial Basis Function) para classificação. Neste trabalho, propõem-se utilizar a abordagem SVM one class, ilustrada pela Fig. 3.

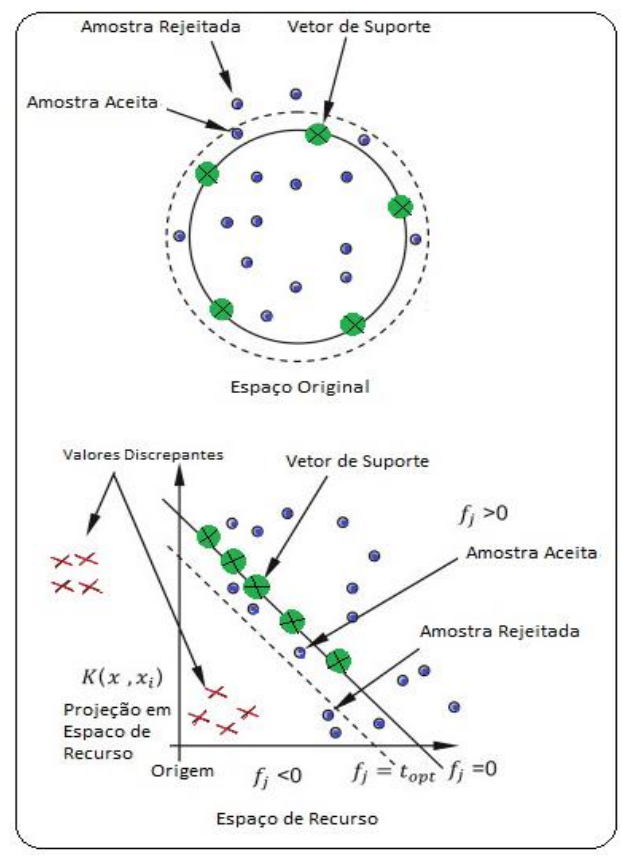

Fig. 3. Modelo do detector baseado em SVM one class.

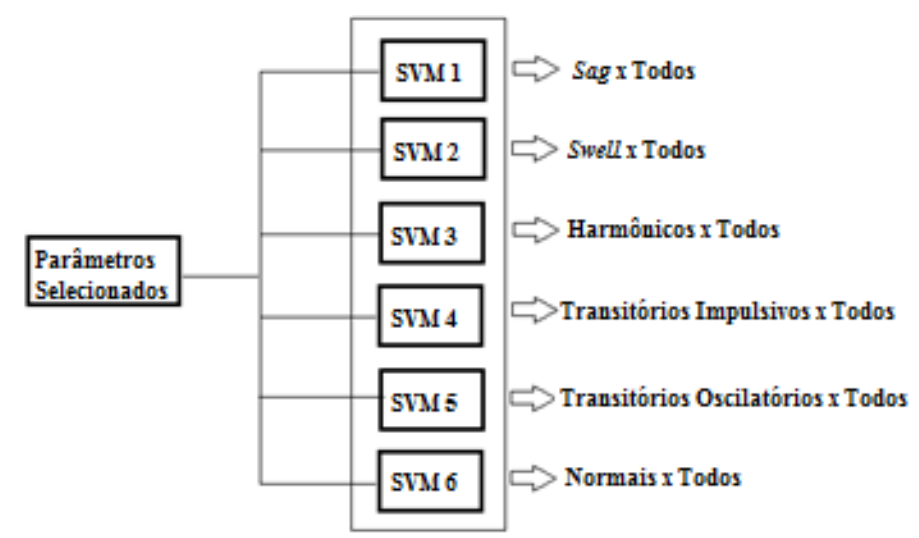

Fig. 4. Sistema de Classificação Especialista baseado em SVM one class. 


\section{RESULTADOS}

Conforme explicado, na metodologia proposta, após a extração dos cumulantes de $2^{\mathrm{a}}, 3^{\mathrm{a}}$ e $4^{\mathrm{a}}$ ordens, aplicou-se o Discriminante Linear de Fisher que possibilitou a apresentação de apenas quinze lags dentre os mais representativos da classe ao seu respectivo classificador especialista baseado em SVM one class.

Os resultados individuais de classificação, para os eventos de teste, são mostrados na Tabela I em relação ao percentual de detecção $(\mathrm{Pd})$ e percentual de falso alarme (Pf), onde pode-se observar que o desempenho individual de cada classificador é satisfatório, com a eficiência se situando acima de $99.9 \%$ considerando-se a média aritmética dos desempenhos obtidos para cada classe de distúrbio. Sendo o percentual de falso alarme, quando o método determina que o distúrbio pertence à classe, entretanto o distúrbio não pertence.

\section{TABELA I DESEMPENHO INDIVIDUAL DOS SISTEMAS DE CLASSIFICAÇÃO ESPECIALISTA EM \%}

\begin{tabular}{lll}
\hline $\begin{array}{l}\text { Sistema de Classificação } \\
\text { Especialista }\end{array}$ & Pd & Pf \\
\hline Afundamento de Tensão & 98.66 & 3.0 \\
Elevação de Tensão & 100.00 & 4.2 \\
Harmônicos & 99.74 & 2.4 \\
Transitório Impulsivo & 100.00 & 4.6 \\
Transitório Oscilatório & 100.00 & 10.8 \\
Normais (Sem distúrbios) & 100.00 & 4.2 \\
\hline
\end{tabular}

A Tabela II mostra a classificação final de acertos dos indivíduos. Para realizar esta avaliação o sinal de teste contaminado um tipo de distúrbio é analisado por todos os classificadores especialistas, e só é considerado como acerto caso a lógica final de classificação indique o sinal como pertencente a sua devida classe de distúrbio e não pertencente as demais. Os resultados apontaram um total de acertos de $97.23 \%$, o que é considerado um bom resultado de classificação.

\section{TABELA II DESEMPENHO GLOBAL DOS SISTEMAS DE} CLASSIFICAÇÃO ESPECIALISTA EM \%

\begin{tabular}{lc}
\hline $\begin{array}{l}\text { Sistema de Classificação } \\
\text { Especialista }\end{array}$ & Acertos \\
\hline Afundamento de Tensão & 97.00 \\
Elevação de Tensão & 95.80 \\
Harmônicos & 99.74 \\
Transitório Impulsivo & 93.80 \\
Transitório Oscilatório & 99.80 \\
\hline
\end{tabular}

\section{CONCLUSÕES}

O presente trabalho propôs um método de detecção e classificação de distúrbios de QEE utilizando ferramentas de reconhecimento de padrões. Foram explorados os cumulantes de segunda, terceira e quarta ordens para extração de parâmetros dos distúrbios. A aplicação do Discriminante
Linear de Fisher resultou em um ganho em redução de dimensão (de 256 parâmetros para 15), a opção pelo método Fisher é devido ao fato de ser um método simples e com bons resultados em pesquisas na área. $\mathrm{O}$ sistema utilizou o método SVM one class, para classificar distúrbios. Para o uso desta ferramenta de classificação é suficiente apenas o conhecimento dos parâmetros de uma das classes em um problema de classificação de duas classes. Baseado nesta característica foi desenvolvido um classificador especialista para cada uma das classes de distúrbios aqui abordadas. Este sistema de classificação apresenta a vantagem de ser possível inserir facilmente uma nova classe de distúrbio a ser classificada, não precisando reformular o classificador. Portanto este tipo de sistema torna-se útil visando as constantes mudanças observadas no cenário dos sistemas de energia elétrica. Os resultados são promissores, com taxas de classificação superiores a $97 \%$.

Em trabalhos futuros espera-se investigar o ganho em desempenho do método proposto com o acréscimo de ferramentas sofisticadas de seleção de parâmetros, como por exemplo Algoritmos Genéticos, visto que esse método tem a capacidade de aperfeiçoar o desempenho dos classificadores. Além disso, pretende-se testar o desempenho do método considerando múltiplos distúrbios.

\section{AGRADECIMENTOS}

Ao Conselho Nacional de Desenvolvimento Científico e Tecnológico (CNPq), à Fundação de Amparo à Pesquisa do Estado de Minas Gerais (FAPEMIG) pelo suporte financeiro.

\section{REFERÊNCIAS}

Commission, I. I. E. IEC-91- Draft Classifcation of Electromagnetic Environments, Technical report, IEC: TC77WG6 (Secretary) 110-R5. 1991.

IEEE Recommended Practice for Monitoring Electric Power Quality, IEEE Std 11592009 (Revision of IEEE Std 11591995) pp. c1-81, 2009.

Nikias, C., Petropulu, A., "HigherOrder Spectra Analysis - A Nonlinear Signal Processing Framework", Englewood Cliffs, Prentice-Hall, 1993.

Ferreira, D., "Power system's electrical disturbance alasysis Análise de aistúrbios elétricos em sistemas de potência", $\mathrm{PhD}$ thesis, Universidade Federal do Rio de Janeiro, Electrical Engineering Doctorship, 2010.

Ferreira, D. D., Cerqueira, A. S., Ribeiro, M. V. and Duque, C. A. (2006). Hos-based method for power quality event classification, in IEEE (ed.), Signal Processing Conference, 2006 14th European, Signal Processing Conference, 2006 14th European, pp. 1-5.

Ferreira, D., Seixas, J., Duque, C., Cerqueira, A., Ribeiro, P., "A direct approach for disturbance detection based on principal curves", in IEEE (ed.), Harmonics and Quality of Power (ICHQP), 2014 IEEE 16th International Conference on, Bucharest, pp. $747-751$.

Tomic, J., Kusljevic, M., Vujicic,V., "A new power system digital harmonic analyzer", IEEE Transactions on Power Delivery 22(2): 772-780, 2007. 
Mendel, J., Tutorial on higher-order statistics (spectra) in signal processing and system theory: Theoretical results and some applications. Proceedings of the IEEE, Ieee, v. 79, n. 3, p. 278-305, 1991.

Theodoridis, S., Pikrakis, A., Koutroumbas, K., Cavouras, D. "Introduction to Pattern Recognition: A Matlab Approach", Academic Press, 2010.

R. Kumar; B. Singh; D. T. Shahani; A. Chandra; K. AlHaddad. Recognition of Power-Quality Disturbances Using STransformBased ANN Classifier and Rule-Based Decision Tree. IEEE Transactions on Industry Applications, vol. 51, n. 2, 2014.

ANEEL - Resolução ANEEL nº 505/2001 Conformidade dos níveis de tensão. Disponível em: http://www2.aneel.gov.br/cedoc/bres2001505.pdf. Acessado em: 27/03/2019 